https://doi.org/10.46344/JBINO.2021.v10i2b.05

\title{
MINI REVIEW ON SELECTION OF CHROMATOGRAPHIC TECHNIQUES FOR THERAPEUTIC DRUG MONITORING
}

\section{Simranjeet Kaur* \& Gourav Kalra}

' Faculty of Pharmaceutical Sciences, PCTE Group of Institutes, Baddowal- 141012, Ludhiana, Punjab, India

\begin{tabular}{|c|}
\hline \\
\hline $\begin{array}{l}\text { Therapeutic drug monitoring (TDM) is an instrument used to incorporate } \\
\text { pharmacokinetic and pharmaco dynamic information to upgrade and customize drug } \\
\text { treatment. TDM is of explicit interest for enemies of denunciations to guarantee sufficient } \\
\text { medication presentation and diminish unfavorable occasions, to build tolerant } \\
\text { consistence and to forestall antimicrobial obstruction. For TDM, drug blood focuses are } \\
\text { resolved to bring and keep the fixation inside the focused on remedial reach. Presently, } \\
\text { LC-MS/MS is the essential scientific strategy for quick and precise measurement of } \\
\text { against infective medication focuses. Notwithstanding blood, a few elective } \\
\text { frameworks (cerebrospinal liquid, incendiary liquids, explicit cells and tissue) and } \\
\text { elective inspecting techniques (dried blood spot and saliva) are right now being } \\
\text { explored. Here, we survey the current difficulties in the bio investigation of hostile to } \\
\text { infective medications and give understanding in the pre-and post-insightful issues } \\
\text { encompassing TDM. }\end{array}$ \\
\hline
\end{tabular}

Keywords: Liquid chromatography, Mass spectroscopy, Therapeutic drug monitoring 


\section{INTRODUCTION}

Traditionally, therapeutic drug monitoring was confined to against epileptic medications and amino glycosides, yet additionally now covers - among others immunosuppressant drugs, drugs following up on the cardiovascular framework, hostile to HIV medications and antifungal medications. For certain classes of medications, TDM has not just demonstrated to be useful for understanding result, yet additionally to be cost-effective ${ }^{5}$ with expanding microbe protection from hostile to infective medications, there is an unmistakable requirement for new specialists.' However, the advancement of new enemy of infective is tedious and costly. Along these lines, treatment enhancement of the current enemy of infective should be a focal point of contemporary treatment. Because of its criticalness, improvement of antimicrobial opposition has a high need for some associations and even entered the political plans. Treatment improvement can be acknowledged by choosing the proper antimicrobial medication, guaranteeing sufficient medication introduction corresponding to the vulnerability of the miniature creature and decreasing antagonistic occasions to build patient's consistence with treatment. For a long time, immunoassays and customary superior fluid chromatography (HPLC) strategies were the significant procedures used to decide centralizations of hostile to infective medications in human examples. ${ }^{2}$ Notwithstanding, immunoassay strategies are just accessible for a predetermined number of medications and crossreactivity, for example with drugs and their metabolites, is an issue. HPLC-UV frequently requires broad example preparation and is in this way work serious. Moreover, long runtimes are frequently needed to get a particular examination technique. ${ }^{3}$ In increases, the two immunoassays and HPLC-UV strategies frequently need affectability. These days, insightful difficulties like these have been overwhelmed with the presentation of HPLC combined with pair mass spectrometry (LC-MS/MS). With the utilization of LC-MS/MS, affectability and selectivity has fundamentally improved, permitting basic and quick example arrangements and short runtimes. ${ }^{4}$ This survey will zero in on the bio logical obstacles identified with the estimation of hostile to infective medications, yet in addition will give understanding in preand post-insightful issues to help clinical scientists, clinical pharmacologists and scientific specialists to increase their expectations. 5

\section{TDM}

For more than 30 years, TDM has been utilized as an instrument to incorporate pharmacokinetic and pharmacodynamic information to enhance drug treatment at the individual patient level. 6,7 Pharmacokinetics portrays the conduct of a medication in the patient's body, including ingestion, dissemination, digestion and discharge, though pharmacodynamics depicts the biochemical or pharmacological impact of a medication on the patient's body or miniature creature inside the body. 
Together, the two boundaries decide the pharmacological profile of the medication. TDM utilizes drug blood focuses to customize drug treatment to bring and keep the fixation inside the focused on restorative reach. Beneath this reach the medication focus is sub restorative or inadequate, though high fixations may bring about antagonistic occasions or poisonousness. TDM is utilized when it is difficult to gauge the pharmacodynamic impact of the medication quicker or in a more straightforward manner, or it is utilized to improve dosing in patients with seriously adjusted pharmacokinetic boundaries (for example basically sick patients in ICU). 8 For hostile to infectives, it is both troublesome and tedious to see whether the disease is being dealt with enough. On the off chance that the disease isn't dealt with satisfactorily, might be past the point where it is possible to reverse the situation of sickness, bringing about therapy disappointment including tolerant dreariness or mortality or the development of antimicrobial obstruction. Before TDM can be played out, a few essentials must be satisfied. Initial, a focus impact relationship or remedial reach should be set up. Besides, enormous entomb individual (for example sex, age or hereditary varieties) or intra singular changeability (for example drug-drug cooperations, diminished renal capacity or liver disappointment) in pharmacokinetics should be noticed, bringing about a huge variety in blood fixations. It plays a significant Use of an Antifungal by utilizing the TDM Service. ${ }^{9}$ The last evident essential is that a touchy and explicit examine should be accessible to decide the medication in blood or other organic grids 9

\section{HPLC IN TDM.}

High performance liquid chromatography (or High pressure liquid chromatography, HPLC) is a particular type of section chromatography by and large utilized in biochemistry and investigation to isolate, recognize, and measure the dynamic compounds.' HPLC mostly uses a segment that holds pressing material (Stationary phase), a pump that moves the mobile phase(s) through the segment, and an identifier that shows the maintenance seasons of the atoms. Maintenance time changes relying upon the cooperations between the fixed stage, the atoms being broke down, and the solvent(s) used. 10 The example to be investigated is acquainted in little volume with the flood of mobile phase and is impeded by explicit substance or actual collaborations with the stationary phase. The measure of impediment relies upon the idea of the analyte and piece of both fixed and portable stage. The time at which a particular analyte elutes (emerges from the finish of the section) is known as the retention time. Basic solvents utilized incorporate any miscible blends of water or natural fluids (the most well-known are methanol and acetonitrile). ${ }^{2}$ '3 Sepration has been done to change the mobile phase arrangement during the investigation; this is known as gradient elution. ${ }^{3}$ The gradient isolates the analyte combinations as a component of the liking of the analyte for the flow mobile phase. The selection of solvents, added substances and gradient rely upon the idea of the stationary phase and the analyte. 


\section{HPLC-UV IN TDM}

HPLC in mix with UV detection is the technique regularly applied for the assurance of most anti-toxins in biological samples. The technique is regularly reasonable for the assurance of antiinfection agents since they are oftentimes utilized in high portions, and the focus accomplished in blood or different tissues is high to such an extent that their evaluation by the HPLC-UV method is conceivable. There are a couple of instances of techniques for examining antifungal medications utilizing UV detection. ${ }^{8}$ HPLC-UV doesn't need the utilization of confounded gear, very much prepared staff, and greater expenses, which makes it conceivable to generally utilize it in clinical labs. ${ }^{11}$

\section{LC-MS/MS IN TDM}

LC-MS/MS has these days set up itself as the essential scientific procedure to help TDM. The regularly utilized lattices for TDM are blood, plasma, and serum and volumetric absorptive microsampling for quantitative bioanalysis of cathinone analogs in dried urine. ${ }^{12}$ All the more as of late, dried blood spots (DBS) and spit have been presented for TDM. Grids like cerebrospinal liquid (CSF), provocative liquids, explicit cells and tissue are not regularly utilized for TDM, however might be important. In any case, every grid has its insightful focal points and weaknesses and the clinical translation of the outcomes unequivocally relies upon this framework. Various rules on bio insightful and clinical strategy approval have been distributed to improve and guarantee the nature of logical technique approval and the created scientific outcomes. Among these are the Food and Medication Organization (FDA) with the 'bioanalytical technique

approval',

European

Prescriptions Office Board (EMEA) with the 'rule on bioanalytical approval', and the Clinical and Research facility Principles Foundation (CLSI) with the 'C62-A, Fluid Chromatography-Mass Spectrometry Strategies; Endorsed Rule'. LC-MS/MS has supplanted HPLC-UV in numerous clinical research centers in major league salary nations. Tragically, the necessary wide collection of antimicrobial medication measures important for an enemy of infective TDM program will lessen the quantity of tests per LC-MS/MS instrument every year, bringing about a generally excessive cost for each test. Albeit less appealing from a research center point of view, costs coming about because of deficient antimicrobial treatment are a lot higher. On the off chance that modest, first-line against infectives fizzle and must be changed to rescue treatment with second-line hostile to infective medications, costs will rise considerably. Before a clinic makes interests in a LC-MS/MS to support a TDM program for antimicrobial medications, one should put forth a business defense. When all is said in done 20.000-50.000 tests yearly are viewed as an adequate avocation of the venture. For little clinics, joining a LC-MS/MS for other TDM programs too for example antidepressants, antipsychotics or immunosuppressants), could bring about practical activity of a LC-MS/MS. LCMS/MS is an application for restorative medication observing in option matrices." Another option could be sending an example to a close by reference focus, if turnaround time is adequate. For low pay nations, HPLC-UV actually is an option as long as affectability isn't an issue. Ideally, 
expanded utilization of LC MS/MS in clinical research facilities will bring about lower speculations costs empowering more extensive execution of LC-MS/MS. ${ }^{13}$

\section{Free dRUG CONCENTRATION}

Regularly, blood concentrations for TDM are determined as total drug concentrations, ${ }^{9}$ i.e. the sum of the unbound and plasma protein bound fraction of the drug. However, only the unbound, free drug can diffuse through biological membranes to the site of action and exert its pharmacological and/or toxicological effects. Therefore for highly protein bound drugs, a small change in the extent of protein binding may result in a major change in free fraction of high protein bound drugs. In clinical practice, unbound drug concentrations of highly protein bound drugs may be relevant for specific conditions, for instance in critically ill patients $^{4}$ suffering from hypo albuminemia. This results in a higher free fraction of that particular drug with subsequently several effects. Initially the unbound drug concentration increases. Since only the unbound drug can be removed from the blood, the amount of drug cleared from the blood increases. Furthermore, the distribution of the unbound drug from the blood to peripheral tissues is increased. As a result, the unbound drug concentration decreases to the original value, while the total drug concentration is decreased. Therefore, total drug concentrations may not be representative for the effective $P K / P^{10}$ index and the unbound drug concentration should be measured instead of total drug concentration, in particular for highly bound drugs.

\section{Proficiency testing PROgRAMme}

An assortment of logical strategies has been distributed for the evaluation of against infective medications in human serum or plasma. The unwavering quality of these scientific strategies is fundamental to give data on the medication fixation to the antimicrobial stewardship that ideally interprets in the best result for our patients. Intra lab strategy approval and intra research center strategies, for example, approval of gear and capability of professionals, ought to guarantee that these techniques have adequate exactness, accuracy and particularity. Support in a bury lab QC or Proficiency Testing (PT) customized is a basic segment of value confirmation and furthermore gives proof of lab ability to clinicians, scientists, certifying bodies and administrative offices. Capability Testing Project are utilized for estimation of antifungal medication concentrations. ${ }^{13}$ A PT modified is fundamental to check whether the scientific technique utilized for TDM follows the quality needed for understanding consideration. Numerous PT programs exist in the field of HIV, antifungal and antituberculosis sedates and have surely prompted scientific improvement. For example, in a PT program for the estimation of antifungal medication fixations, the outcomes demonstrated that one out of five estimations was off base. The performing research facility was the principle deciding component for these mistakes, which presumably implies that intra lab technique approval was wrong. Moreover, the consequences of a PT program for antiretroviral drugs indicated that the estimation of low antiretroviral fixations likewise was dangerous and 
prompted wrong dosing proposals. These models show and underline the significance of PT programs for scientific strategies utilized for TDM in clinical practice. ${ }^{14}$ Outpatient checking regularly, blood tests is utilized for TDM which are frequently gathered by vena cut. In any case, this testing system has a few hindrances. To start with, venous examining is troublesome in certain populaces, for example, youngsters and patients experiencing venous harm. Second, there might be strategic difficulties. For venous testing the patient necessities to head out to the clinic or an assigned lab. This may not generally be conceivable, for example in asset restricted and far off territories. Another issue, particularly in (sub) tropical territories, is test security. Numerous medications are not steady in serum or plasma at room temperature and must be put away and moved at - $20{ }^{\circ} \mathrm{C}$ or lower. To determine these soundness issues, elective examining techniques have been grown, for example, DBS, dried plasma spots and miniature testing. DBS inspecting is progressively applied for advancing medication measurements for some medications. DBS is famous for its favorable circumstances like negligible obtrusive examining, test dependability and little blood volume needed for examination. All in all, a DBS test comprises of a fringe blood test got by a finger prick. With clear guidelines and in the wake of preparing, patients will have the option to play out the method themselves at home. DBS techniques have been distributed for a few antibacterial, antifungal and antiretroviral drugs. Reference esteems for TDM are customarily founded on serum or plasma drug fixations and not on entire blood focuses. Accordingly, clinical approval is needed to make an interpretation of hairlike blood-to-serum or plasma concentration. Another conceivable significant factor might be the cooperation of the medication with the blood lattice or the DBS card grid. Rifampicin has shown to interface with endogenous blood parts, as ferric particles from the red platelets causing complex development. This causes low recuperations from DBS removes which can be improved by the expansion of chelating specialists, for example, EDTA and deferoxamine, to the extraction methodology. ${ }^{14}$ Additionally immediate official of the medication by hydrogen holding with the DBS card lattice may affect recuperation. Recuperation additionally is impacted by haematocrit esteem, substance focus and drying season of the DBS card. This collaboration is inborn to the current cellulose based card networks. A bit of leeway of the dried plasma spot procedure over DBS is that it isn't affected by haematocrit value. Quantification against infective medications utilizing the dried plasma spot strategy has been depicted for fosfomycin, daptomycin, linezolid, triazole antifungal medications and antiretroviral drugs. Despite the fact that the utilization of DBS and dried plasma spot methods isn't yet broadly spread, both are a promising option for venous blood examining and now and again (for example low asset and far off regions) the solitary feasible alternatives. Another patient agreeable strategy for inspecting is the utilization of salivation. Contrasted with blood testing, salivation is anything but difficult to gather and non-obtrusively with an irrelevant possibility of contaminations. Moreover, it is modest 
and makes less pressure and inconvenience the patients. As spit is a low protein lattice ( $0.3 \%)$, the deliberate focuses speaks to the unbound grouping of the medication. This may require a delicate LC-MS/MS ${ }^{15}$ investigation technique or a broad example readiness system like SPE or LLE to focus the example for drugs with high protein authoritative. As there are numerous different determinants of the salivary medication focus, for example, salivary stream rate, steadiness of the medication and its metabolites, season of test assortment and ingestion of food or refreshments target fixations in salivation should be set up on a medication tosedate premise Spit strategies utilizing LCMS/MS have been distributed for a couple of against infective medications (doxycycline, fluconazole, linezolid, lopinavir and oseltamivir)

\section{CONCLUSION}

Taking everything into account, TDM assumes a significant part in the improvement of treatment with against infective medications. To perform TDM satisfactorily, it is fundamental to configuration measures with a fast turnaround time, empowering the antimicrobial stewardship to rapidly change and improve treatment if important. LC-MS/MS is a quick and precise strategy for evaluation of against infective medications. On the off chance that a logical strategy is created and approved, bury research center quality control is a significant segment of value affirmation. In clinical practice blood is the most usually utilized framework for TDM since it fills in as a decent proxy for the site of contamination. As a rule, it is effortlessly gotten, rather than different lattices. Be that as it may, in complex irresistible cases different lattices could be utilized to advance enemy of infective treatment.

\section{References}

1. Martin M., Guiochon, G. Effects of high pressures in liquid chromatography. J. Chromatogr. A, 2005; (1-2)7: 16-38.

2. Liu Y., Lee M.L. Ultrahigh pressure liquid chromatography using elevated temperature. Journal of Chromatography. 2006; 1104 (1-2): 198-202.

3. Abidi,S.L. High-performance liquid chromatography of phosphatidic acids and related polar lipids. J. Chromatogr. 1991; 587: 193-203.

4. J.A. Roberts, M.H. Abdul-Aziz, J. Lipman, J.W. Mouton, A.A. Vinks, T.W. Felton, et al., Individualised antibiotic dosing for patients who are critically ill: challenges and potential solutions., Lancet. Infect. Dis.

5. D.J. Touw, C. Neef, A.H. Thomson, A.A. Vinks, C.-E. of Therapeutic Drug Monitoring Committee of the International Association for Therapeutic Drug Monitoring, C. Toxicology, Cost-effectiveness of therapeutic drug monitoring: a systematic review, Ther. Drug Monit. 27(2005) 10-17.

6. R.J.M. Brüggemann, R.E. Aarnoutse, Fundament and Prerequisites for the 551 Application of an Antifungal TDM Service. Curr. Fungal Infect. Rep. 9

7. R.E. Aarnoutse, J.M. Schapiro, C.A.B. Boucher, Y.A. Hekster, D.M. Burger, Therapeutic drug monitoring: an aid to optimising 
response to antiretroviral drugs? Drugs 63 (2003)

8. A.F. van der Meer, M.A. Marcus, D.J. Touw, J.H. Proost, C. Neef, analyzing antifungal drugs using UV detection 'HPLC-UV'., Ther. Drug Monit. 33 (2011) 133-46.

9. J.W. Mouton, P.G. Ambrose, R. Canton, G.L. Drusano, S. Harbarth, A. MacGowan, et al., determined as total drug concentrations., Drug Resist. Updat. 14 (2011) 107-17.

10.S. Schmidt, A. Barbour, M. Sahre, K.H. Rand, H. Derendorf, PK/PD: new 564 index and the unbound drug concentration, Curr. Opin. Pharmacol. 8565 (2008) .

11. Avataneo V, D'Avolio A, Cusato J, Cantù $M$, De Nicolò A. LC-MS application for therapeutic drug monitoring in alternative matrices. J Pharm Biomed Anal. 2019 Mar 20; 166:40-51.

12. Mercolini L, Protti M, Catapano MC, Rudge J, Sberna AE. LC-MS/MS and volumetric absorptive microsampling for quantitative bioanalysis of cathinone analogues in dried urine, plasma and oral fluid samples. J Pharm Biomed Anal. 2016 May

13. V. J. C. Lempers, J. W. C. Alffenaar, D. J. Touw, D. M. Burger, D. R. A. Uges, R. E. Aarnoutse, R. J. M. Brüggemann, Five year results of an international proficiency testing programme for measurement of antifungal drug concentrations, Journal of Antimicrobial Chemotherapy, Volume 69, Issue 11. November 2014, Pages 29882994

14. Ashbee HR, Barnes RA, Johnson EM, Richardson MD, Gorton R, Hope
WW. Therapeutic drug monitoring (TDM) of antifungal agents: guidelines from the British Society for Medical Mycology. J Antimicrob Chemother 2014;69:1 162-76.

15. Pauwels S, Vermeersch $P$, Van Eldere J, Desmet K. Fast and simple LC-MS/MS method for quantifying plasma voriconazole. Clin Chim Acta 2012;413:740-3.

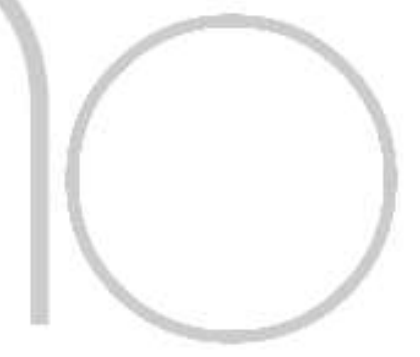

Gallery Proof copy 